\title{
On Solutions of Initial-Boundary Problem
}

$$
\text { for } u_{t}=u_{x x}+\frac{1}{1-u}
$$

\section{Hideo KAWARADA*}

\section{$\S 1$. Introduction and Theorem}

Various works [1], [2], [3] have been published on the blowingup of solutions of the Cauchy problem and the initial-boundary value problem of nonlinear partial differential equations. Blowing-up means that the solutions of these problems bccome infinite in a finite time.

The objective of the present paper is to introduce the concept of quenching which has more general sense than blowing-up and to find some sufficient conditions for quenching of the solutions of the following initial-boundary value problem for $u=u(t, x), t>0, x \in(0, l)$,

$$
\begin{array}{ll}
\text { (1.1a) } & u_{t}=u_{x x}+\frac{1}{1-u}, \quad t>0, \quad x \in(0, l), \\
(1.1 \mathrm{~b}) & u(t, 0)=u(t, l)=0, \quad t>0, \\
(1.1 \mathrm{c}) & u(0, x)=0, \quad x \in(0, l),
\end{array}
$$

where $l$ is a positive constant. The above initial-boundary value problem $(1.1 \mathrm{a} \sim \mathrm{c})$ is denoted by IVP. Our study may be said to be more illustrative than general, since we restrict ourselves to mixed problems of semilinear heat equations with space dimension one. Nevertheless, we hope that our results will give an insight into a more general situation. The nonlinear perturbation $\frac{1}{1-u}(u \neq 1)$ in $(1.1 \mathrm{a})$ is locally Lipschitz continuous. Thus IVP has a unique solution which may be local in $t$.

Communicated by S. Hitotumatu, July 26, 1974.

* Department of Applicd Physics, University of Tohyo, Tokyo. 
The present problems came to our attention in connection with the diffusion equation generated by a polarization phenomena in ionic conductors [4].

We shall define quenching for the solutions of the initial value problems.

Definition 1. Let $u=u(t, x)$ be the solution of the initial value problems which are defined in $t>0, x \in \Omega$. $\Omega$ means $R^{m}$ which stands for the $m$-dimensional Euclidean space or the bounded domain in $R^{m}$.

We shall say that $u$ quenches if $\left\|u_{t}\right\|_{c}$ becomes infinite in a finite time where $\|\cdot\|_{c}$ denotes the maximum norm over $\Omega$.

In order to clarify the nature of quenching, let us take some examples.

Example 1. $\alpha$ being constant, the solution of the initial value problem for $u=u(t), t>0$,

$$
\left\{\begin{array}{l}
\frac{d u}{d t}=\frac{1}{1-u}, \quad t>0 \\
u(0)=\alpha,
\end{array}\right.
$$

is $u=1+\sqrt{(1-\alpha)^{2}-2 t}$, if $\alpha>1$ and $u=1-\sqrt{(1-\alpha)^{2}-2 t}$, if $\alpha<1$. In both cases, we see quenching at $t=\frac{(1-\alpha)^{2}}{2}$.

Example 2. Let $\alpha$ be as above. The solution of the initial-boundary value problem for $u=u(t, x), t>0, x \in(0, l)$,

$$
\left\{\begin{array}{l}
u_{t}=u_{x x}+\frac{1}{1-u}, \quad t>0, \quad x \in(0, l) \\
u_{x}(t, 0)=u_{x}(t, l)=0, \quad t>0 \\
u(0, x)=\alpha, \quad x \in(0, l)
\end{array}\right.
$$

is the same as above.

Example 3. Blowing-up in the initial value problems means quenching. 
As our main result, we have

Theorem. In the IVP, suppose $l>2 \sqrt{2}$. Then the solution of the IVP quenches.

The present paper has two sections apart from this section. In $\S 2$, we shall give a Lemma. $\$ 3$ is devoted to the proof of our Theorem.

\section{§2. Lemma}

As a preparation for the proof of Theorem we state the following lemma. Henceforce, let $u=u(t, x)$ be the solution of IVP.

Lemma. In the IVP, suppose $l>2 \sqrt{2}$. Then $u$ reaches 1 in a finite time at $x=\frac{l}{2}$.

Proof:

1st Step. We show that $u(t, x)$ is increasing in $t$ for every $x$ in $(0, l)$ as long as $u$ exists. In fact, putting $v=u_{t}$, we have

$$
\begin{aligned}
v_{t} & =v_{x x}+\frac{1}{(1-u)^{2}} \cdot v, \quad x \in(0, l), \\
v(t, 0) & =v(t, l)=0
\end{aligned}
$$

and

$$
v(0, x)=1, \quad x \in(0, l) \quad \text { as long as } u \text { exists }
$$

We notice that $v$ is a solution of the linear parabolic equation (2.1) and is non-negative on the "parabolic boundary". Thus $v$ is nonnegative everywhere, which implies the required monotonicity of $u$.

2nd Step. The solution $u_{1}=u_{1}(t, x)$ of the initial-boundary value problem for $u=u(t, x)$,

$$
\begin{cases}u_{t}=u_{x x}+1, & t>0, \quad x \in(0, l), \\ u(t, 0)=u(t, l)=0, & t>0, \\ u(0, x)=0, & x \in(0, l)\end{cases}
$$


converges its stationary solution $\psi(x)=\frac{1}{2} l(l-x)(0<x<l)$ as $t \rightarrow+\infty$. Thus $u_{1}$ crosses 1 in a finite time if $l>2 \sqrt{2}$.

Suppose that $u$ does not reach 1 in a finite time if $l>2 \sqrt{2}$. Then IVP has a global solution, i.e., $u$ satisfies $0 \leqq u \leqq 1$ in $(0, l) \times[0,+\infty)$ by virtue of the monotonicity of $u$. Comparing $u$ with $u_{1}$, we get $u \geqq u_{1}$ in $(0, l) \times[0,+\infty)$ since $\frac{1}{1-\lambda} \geqq 1$ in $0 \leqq \lambda \leqq 1$. This contradicts the assumption. We shall denote the time when $u$ reaches 1 by $t=T_{0}$.

3rd Step. $u$ satisfies (i) $u_{x}(t, 0)>0$ by virtue of positivity of $u$; (ii) $u_{x}\left(t, \frac{l}{2}\right)=0$ since $u$ is symmetric with respect to $x=\frac{l}{2}$. Putting $\pi=$ $u_{x}$, we have

$$
\begin{aligned}
& \pi_{t}=\pi_{x x}+\frac{1}{(1-u)^{2}} \cdot \pi, \quad t \in\left[0, T_{0}\right), \quad x \in\left(0, \frac{l}{2}\right), \\
& \pi(t, 0)>0, \quad \pi\left(t, \frac{l}{2}\right)=0, \quad t \in\left[0, T_{0}\right),
\end{aligned}
$$

and

$$
\pi(0, x)=0, \quad x \in\left(0, \frac{l}{2}\right)
$$

Repeating the same argument as in 1 st Step, we see that

$$
\pi=u_{x}(t, x)>0, \quad t \in\left[0, T_{0}\right), \quad x \in\left(0, \frac{l}{2}\right)
$$

Combining (2.2) and (ii), we get that $u$ takes its maximum at $x=\frac{l}{2}$ for any $t \in\left[0, T_{0}\right)$. This completes the proof.

\section{§3. Proof of Theorem}

\section{1st Step}

1. a) Put $\mu=\mu(t)=u\left(t, \frac{l}{2}\right)$ in $\left[0, T_{0}\right) . \quad \mu$ satisfies

$$
\frac{d \mu}{d t} \leqq \frac{1}{1-\mu} \quad \text { in } \quad\left[T_{0}-\varepsilon, T_{0}\right)
$$


for sufficiently small $\varepsilon(>0)$ since $u_{x x}\left(t, \frac{l}{2}\right) \leqq 0$ in $\left[0, T_{0}\right)$. Put $T_{1}=T_{0}-\varepsilon$ and $\Omega_{\varepsilon}=(0, l) \times\left[T_{1}, T_{0}\right)$. Comparing $\mu(t)$ with $v=v(t)=1-\sqrt{2} \sqrt{T_{0}-t}$ in $\left[T_{1}, T_{0}\right)$, we get

$$
\mu \geqq v, \quad \text { in } \quad\left[T_{1}, T_{0}\right)
$$

since $v$ satisfies (see Example 1)

$$
\frac{d v}{d t}=\frac{1}{1-v}, \quad t \in\left[T_{1}, T_{0}\right)
$$

and

$$
\lim _{t \rightarrow T_{0}} v(t)=1
$$

(3.2) implies that there exists the domain $D_{\varepsilon}$ in which $u$ satisfies

$$
u(t, x) \geqq v(t)
$$

Denote the complement of $D_{\varepsilon}$ by $E_{\varepsilon}$ and put $E_{\varepsilon}^{(1)}=E_{\varepsilon} \cap\left\{\left(0, \frac{l}{2}\right) \times\left[T_{1}, T_{0}\right)\right\}$ and $E_{\varepsilon}^{(2)}=E_{\varepsilon} \cap\left\{\left(\frac{l}{2}, l\right) \times\left[T_{1}, T_{0}\right)\right\}$. For $D_{\varepsilon}$, there may be two cases:

Case (a) $D_{\varepsilon}$ has no interior points, i.e., there holds

$$
u_{x x}\left(t, \frac{l}{2}\right)=0 \quad \text { in }\left[T_{1}, T_{0}\right)
$$

Case (b) $\quad D_{\varepsilon}$ has interior points.

For the case (a), $u$ quenches obviously. Henceforce we consider only the case (b).

1.b) Denote the boundary between $D_{\varepsilon}$ and $E_{\varepsilon}^{(i)}$ by $x=s^{(i)}(t)\left(t \in\left[T_{1}, T_{0}\right)\right)$ for $i=1,2$. Then $x=s^{(i)}(t)$ satisfies

(i) $\lim _{t \rightarrow T_{0}} s^{(i)}(t)=\frac{l}{2}$;

(ii) $u_{x}\left(t, s^{(i)}(t)\right) \cdot \dot{s}^{(i)}(t)=-u_{x x}\left(t, s^{(i)}(t)\right), t \in\left[T_{1}, T_{0}\right)$ where $\dot{s}^{(i)}(t)$ means $\frac{d s^{(i)}(t)}{d t}$ for $i=1,2$. In fact, there holds 


$$
u=v \quad \text { on } \quad x=s^{(i)}(t), \quad t \in\left[T_{1}, T_{0}\right)
$$

Differentiating both sides of (3.3) and using (3.3), we get

$$
u_{t}\left(t, s^{(i)}(t)\right)+u_{x}\left(t, s^{(i)}(t)\right) \cdot \dot{s}^{(i)}(t)=\frac{1}{1-u\left(t, s^{(i)}(t)\right)} .
$$

By virtue of (1.1 a) on $x=s^{(i)}(t)$ and (3.3) we have (ii).

1.c) Obviously we have the following inequalities

$$
\frac{1}{1-u} \geqq \frac{1}{\sqrt{2} \sqrt{T_{0}-t}} \quad \text { in } D_{\varepsilon},
$$

and

$$
\frac{1}{1-u}<\frac{1}{\sqrt{2} \sqrt{T_{0}-t}} \quad \text { in } E_{\varepsilon}
$$

\section{2nd Step.}

2.a) Let $p=p(t, x)$ be $\frac{1}{2\left(T_{0}-t\right)}$ in $D_{\varepsilon}$ and $\frac{1}{(1-u)^{2}}$ in $E_{\varepsilon}$. Then the solution $v_{1}=v_{1}(t, x)$ of the initial-boundary value problem for $v=v(t, x)$ in $\Omega_{\varepsilon}$,

$$
\left\{\begin{array}{l}
v_{\imath}=v_{x x}+p \cdot v \quad \text { in } \Omega_{\varepsilon} \\
v(t, 0)=v(t, l)=0, \quad t \in\left[T_{1}, T_{0}\right), \\
v\left(T_{1}, x\right)=\beta(x)=u_{t}\left(T_{1}, x\right)>0, \quad x \in(0, l),
\end{array}\right.
$$

exists and satisfies $0<v_{1} \leqq v$ in $\Omega_{\varepsilon}$ by virtue of $(3.5 \mathrm{a})$ and the maximum principle (cf. 1st Step in the proof of Lemma).

2. b) Put $W=W(t, x)=\sqrt{T_{0}-t} \cdot v_{1}$. Denoting $W$ in $D_{\varepsilon}$ by $W^{(1)}$, we have $W_{t}^{(1)}=W_{x x}^{(1)}$ in $D_{\varepsilon}$. Furthermore it should be noted that $W(t, x)>0$ in $\Omega_{\varepsilon}$ 。

\section{3rd Step.}

3.a) We shall deal with the following initial-boundary value problem for $V=V(t, x)$ in $(-\infty,+\infty) \times\left[T_{1}, T_{0}\right)$.

$$
V_{t}=V_{x x} \text { in }(-\infty,+\infty) \times\left[T_{1}, T_{0}\right)
$$




$$
\begin{aligned}
& V=W^{(1)} \quad \text { in } \quad D_{\varepsilon} \\
& V=\sqrt{\varepsilon} \cdot \beta(x), \quad x \in\left[0, s^{(1)}\left(T_{1}\right)\right) \cup\left(s^{(2)}\left(T_{1}\right), l\right] \\
& V=0, \quad x \in(-\infty, 0) \cup(l,+\infty) .
\end{aligned}
$$

In what follows we impose on the solution $V(t, x)$ the following conditions at infinity: $V(t, x)$ and $V_{x}(t, x)$ are bounded as $x \rightarrow \pm \infty$ uniformly with respect to $t$ in $\left[T_{1}, T_{0}\right)$. We see the solution $\hat{W}=\hat{W}(t, x)$ of (3.6) uniquely exists. Uniqueness of $\hat{W}$ is shown by Holmgren's theorem. Using the Green's function

$$
K(t, x ; \tau, \xi)=\frac{1}{2 \sqrt{\pi(t-\tau)}} \exp \left\{-\frac{(x-\xi))^{2}}{4(t-\tau)}\right\},
$$

$\hat{W}$ is represented by

$$
\begin{aligned}
\hat{W}(t, x)= & \int_{T_{1}}^{t}\left[K\left(t, x ; \tau, s^{(1)}(\tau)\right) W_{\xi}^{(1)}\left(\tau, s^{(1)}(\tau)\right)\right. \\
& \left.-W^{(1)}\left(\tau, s^{(1)}(\tau)\right) K_{\xi}\left(t, x ; \tau, s^{(1)}(\tau)\right)\right] d \tau \\
& +\int_{0}^{s^{(1)}\left(T_{1}\right)} K\left(t, x ; T_{1}, \xi\right) \sqrt{\varepsilon} \cdot \beta(\xi) d \xi \\
& +\int_{T_{1}}^{t} K\left(t, 0 ; \tau, s^{(1)}(\tau)\right) W^{(1)}\left(\tau, s^{(1)}(\tau)\right) \cdot \dot{s}^{(1)}(\tau) d \tau, \\
& -\infty<x<s^{(1)}(t), t \in\left[T_{1}, T_{0}\right) .
\end{aligned}
$$

Also in $s^{(2)}(t)<x<+\infty, t \in\left[T_{1}, T_{0}\right)$, we have the similar expression as (3.7).

(3.b) Using the positivity of $\beta, W$ and maximum principle, we have

$$
\hat{W}(t, x) \geqq 0 \quad \text { in } \quad(-\infty,+\infty) \times\left[T_{1}, T_{0}\right) .
$$

Thus from (3.6) and (3.5b) we see

$$
\hat{W}(t, x) \geqq W(t, x)>0 \quad \text { in } \quad \Omega_{\varepsilon} .
$$

4th Step. We claim that

$$
\lim _{t \rightarrow T_{0}} \hat{W}\left(t, \frac{l}{2}\right)>0 \text {. }
$$


On the contrary, we suppose that

$$
\lim _{t \rightarrow T_{0}} \hat{W}\left(t, \frac{l}{2}\right)=0
$$

which implies that $0 \equiv \hat{W}(t, x) \geqq W(t, x) \geqq 0$ in $\Omega_{\varepsilon}$ by the strong maximum principle [5]. This is a contradiction. Thus we get that

$$
\lim _{t \rightarrow T_{0}} \frac{d \mu(t)}{d t}=\lim _{t \rightarrow T_{0}} v\left(t, \frac{l}{2}\right) \geqq \lim _{t \rightarrow T_{0}} v_{1}\left(t, \frac{l}{2}\right)=\lim _{t \rightarrow T_{0}} \frac{\hat{W}\left(t, \frac{l}{2}\right)}{\sqrt{l-T_{0}}}=+\infty
$$

This completes the proof.

\section{Acknowledgements}

This paper is dedicated to Professor Isao Imai in celebration of his sixtieth birthday.

\section{References}

[1] Fujita, H., On Some Noncxistence and Nonuniqueness Theorens for Nonlinear Parabolic Equations, Proc. Sympos. Appl. Math., 18 Amer. Math. Soc., Providence, R. I, (1968), 105.

[2] Keller, J. B., On Solutions of Nonlinear Wave Equations, Comm. Pune Appl. Math. 10 (1957), 523.

[3] Kawarada, H., On Solutions of Nonlinear Wave Equations, J. Phys. Soc. Japan, 26 (1971), 280.

[4] Yanagida, H. and Kawarada, H., Mathematical Representation of Electric Current Transient in Polarized Ionic Conductors, to appear.

[5] Friedman, A., Partial Differential Equations of Parabolic Type. Prentice-Hall, Englewood Clifs, N. J., 1964. 